Supporting information for

\title{
A Vernonia Diacylglycerol Acyltransferase Can Increase Renewable Oil Production
}

Tomoko Hatanaka, William Serson, Runzhi Li, Paul Armstrong, Keshun Yu, Todd Pfeiffer, Xi-Le

Li, David Hildebrand*

*Corresponding author

University of Kentucky, Lexington, KY USA

Phone: $859 / 218-0760$

Fax: 859/257-7125

Email: dhild@uky.edu 


\section{Legends in Supporting information}

Figure S1 Lipid contents of transfected Sf9 cells expressing VgDGAT1A, El DGAT1A and the control.

Figure S2 Phylogenetic relationships of soybean, Vernonia, Euphorbia and Arabidopsis DGAT1s with other most related DGAT1s. Bootstrap values are shown on the corresponding places. See Table S1 for their GenBank accession numbers.

Figure S3. Amino acid sequence alignment comparing Vernonia VgDGAT1s, soybean GmDGAT1s and GmDGAT1B-MOD (Roesler et al. 2016) to Arabidopsis AtDGAT1.

Table S1 GenBank accession numbers of DGAT1 genes.

Table S2 Descriptive statistics for the major plant oils and petroleum price trends.

Econometric analysis of the price trends of the major plant oils vs. crude oil (petroleum) in the past 15 years

Table S3 Augmented Dickey-Fuller (ADF) test results for the major plant oils and 
petroleum price.

Table S4 Johansen's test.

Table S5 Results of Granger causality test for the major plant oil and petroleum prices. 


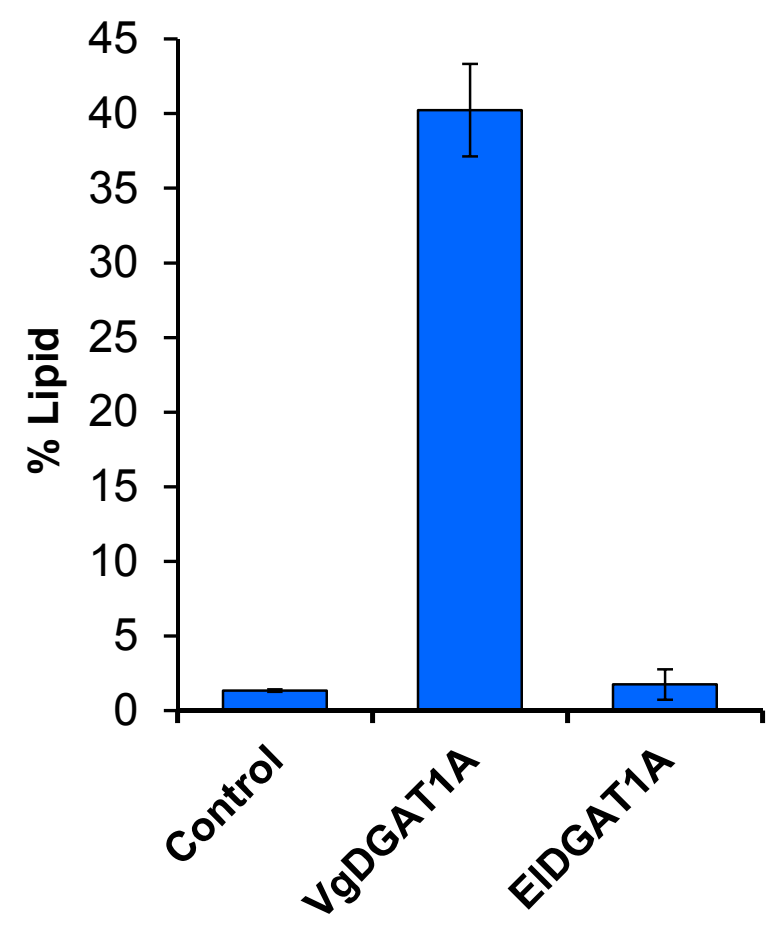

Figure S1.

Transgenes were introduced using the Bac-to-Bac expression system (Gibco BRL) following the manufacturer's recommendations. The cells were infected by baculovirus without cloned genes (Control) or with VgDGAT1A or ElDGAT1A constructs, cultured for four days and had lipids extracted and analyzed for total lipids via GC/MS. ElDGAT1A showed no significant difference from the control in ANOVA analysis $(p=0.9936)$, but VgDGAT1A was significantly higher $(\mathrm{p}=0.0002)$. Error bars are one standard error from the mean for three replicates. 


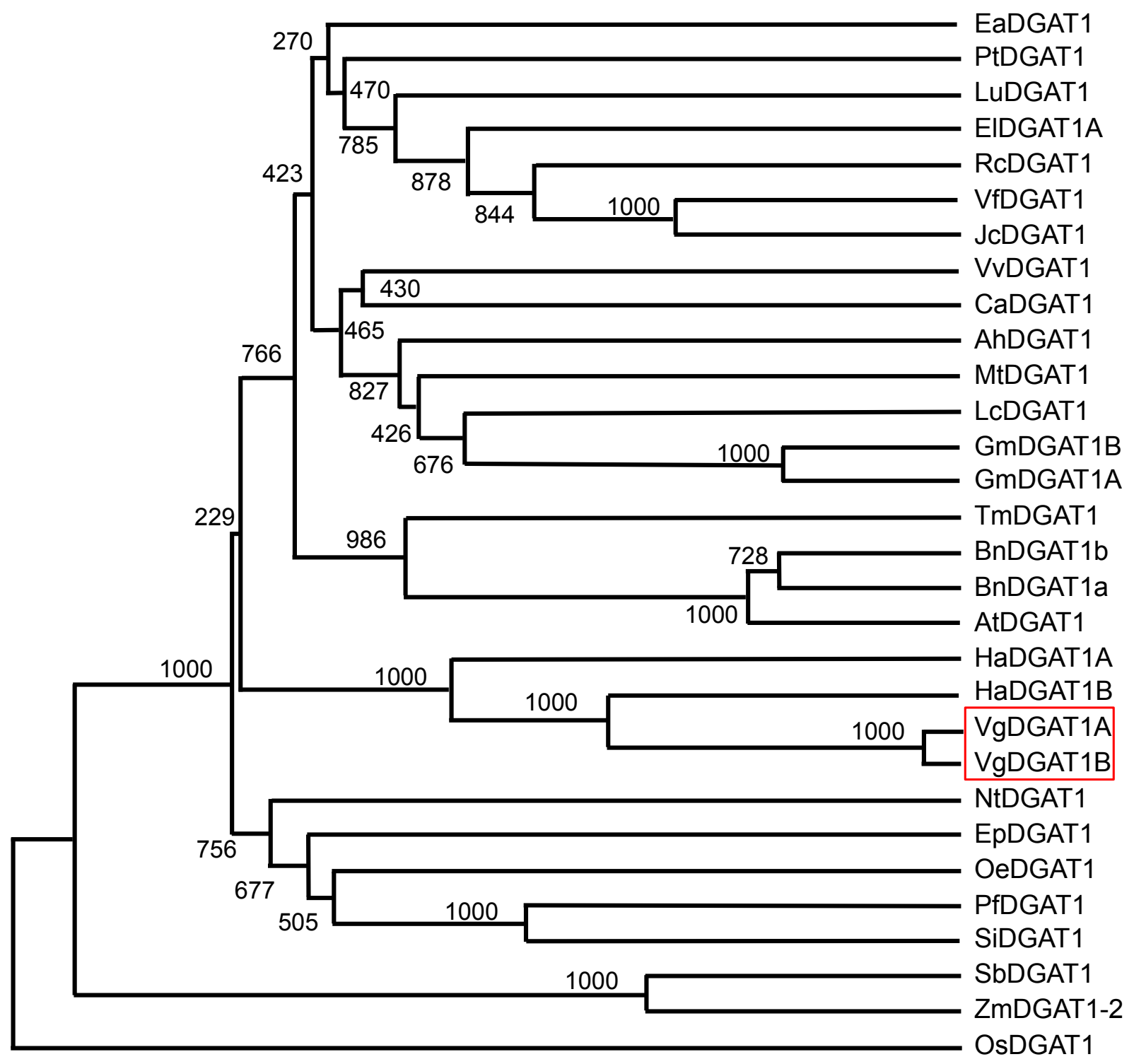

Figure S2. 
Table S1. GenBank accession numbers of DGAT1 genes.

\begin{tabular}{|c|c|c|}
\hline Gene name & Species & Accession Number \\
\hline$A h D G A T 1$ & Arachis hypogaea & KC736068 \\
\hline AtDGAT1 & Arabidopsis thaliana & AJ131831 \\
\hline BnDGATla & Brassica napus & JN224474 \\
\hline$B n D G A T 1 b$ & Brassica napus & JN224476 \\
\hline CaDGAT1 & Corylus americana & KU744408 \\
\hline EaDGAT1 & Euonymus alatus & AY751297 \\
\hline ElDGAT1A & Euphorbia lagascae & AB853120 \\
\hline EpDGAT1 & Echium pitardii & FJ226588 \\
\hline GmDGAT1A & Glycine $\max$ & AB257589 \\
\hline$G m D G A T 1 B$ & Glycine $\max$ & AB257590 \\
\hline HaDGAT1A & Helianthus annuus & EU543809 \\
\hline HaDGAT1B & Helianthus annuus & EU232721 \\
\hline$J_{c D G A T 1}$ & Jatropha curcas & DQ278448 \\
\hline$L c D G A T 1$ & Lotus corniculatus & AY859489 \\
\hline LuDGAT1 & Linum usitatissimum & KC485337 \\
\hline$M t D G A T 1$ & Medicago truncatula & XM_003595183 \\
\hline$N t D G A T 1$ & Nicotiana tabacum & AF129003 \\
\hline OeDGAT1 & Olea europaea & AY445635 \\
\hline OsDGAT1 & Oryza sativa & AY858584 \\
\hline PfDGAT1 & Perilla frutescens & AF298815 \\
\hline PtDGAT1 & Populus trichocarpa & EU477378 \\
\hline RcDGAT1 & Ricinus communis & EU391591 \\
\hline$S b D G A T 1$ & Sorgham bicolor & XM_002437120 \\
\hline SiDGAT1 & Sesamum indicum & JF499689 \\
\hline$T m D G A T 1$ & Tropaeolum majus & AY084052 \\
\hline VfDGAT1 & Vernicia fordii & DQ356680 \\
\hline $\operatorname{VgDGAT1A}$ & Vernonia galamensis & EF653276 \\
\hline$V g D G A T 1 B$ & Vernonia galamensis & EF653277 \\
\hline$V v D G A T 1$ & Vitis vinifera & XM_002279309 \\
\hline ZmDGAT1-2 & Zea mays & EU039830 \\
\hline
\end{tabular}




\begin{tabular}{|c|c|c|c|}
\hline GmDGAT1A & 1 & MA- - - - - - - - - - - - ISDEPETVATAL-NHSS - - IRRRPTAA- - GLENSPETTT & 36 \\
\hline GmDGAT1B & 1 & MA & 39 \\
\hline GmDGAT1B-MOD & 1 & A & 9 \\
\hline $\operatorname{VgDGATIA~}$ & 1 & IALDTPQIGEITTTATTTIRRRTTVKPDAGIGD---G-IFDSSSSSKTNS & 6 \\
\hline VgDGAT1B & 1 & IALITPQIGEITTTATTTIRQHPLGKPDAGIGD---G-IFSSSS: & 5 \\
\hline AtDGAT1 & 1 & IAILDSAGVTTVTENGGGEFV & 0 \\
\hline GmDGAT1A & 37 & SSGDDLAKDSGSDDSISSDAANSQPQ--QKQ---DTDF-SVLKF & 0 \\
\hline GmDGAT1B & 0 & SSGDDLAKDSGSDDSINSDDAAVNSQ--QQNEKQDTDE-SVLKF & 6 \\
\hline GmDGAT1B-MOD & 40 & SSGDDLAKDSGSDDSINNDDAAV & 6 \\
\hline $\operatorname{VgDGATIA}$ & 57 & KGNGQKIDHGGVKKGRET-TVVHYAYRPS|SPAHRIKES & \\
\hline $\operatorname{VgDGAT} 1 \mathrm{~B}$ & 56 & 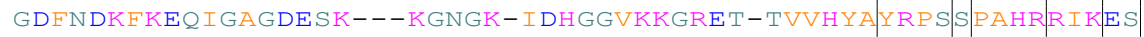 & \\
\hline AtDGAT 1 & 61 & DRIDSVVNDDAQGTANIAGDNNGGGDNNGGGRGGGEGRGNADATFTYRPSVEAHRRARES & \\
\hline mDGAT $1 \mathrm{~A}$ & 1 & QSHAGLFNLCIV & \\
\hline GmDGAT1B & 97 & PLSSDTIFRQSHAGLFNLCIV & 56 \\
\hline GmDGAT1B-MOD & 97 & PLSSDTIFRSHAGLFNLCIV & \\
\hline VgDGAT1A & 116 & NGRIIENLMKYGIIINSNEWES|SR/SLRDWPIIM & \\
\hline $\operatorname{VgDGATIB}$ & 11 & 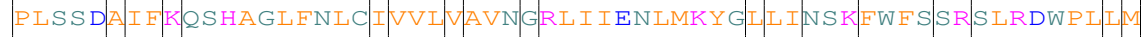 & 70 \\
\hline AtDGAT1 & 21 & PLSSDAIFKQSHA GLFNLCVUVIIAVNSRIIIENLMKYGWLIRTDEWFSSR SIRDWPIFM & \\
\hline GmDGAT1A & 51 & LHIITSSASLFY & \\
\hline GmDGAT1B & 57 & LHIIITSTSLFYE & \\
\hline GmDGAT1B- & 57 & VLHIIITSTSLFYP & \\
\hline VgDGAT1A & 76 & VVITLHVIITTTAIIYPVFMIIREDSVVISGVS & \\
\hline VgDGATIB & 171 & VVITLHVVITTTAIIYPIFMIIREDSVVLIGVS & \\
\hline AtDGAT 1 & 81 & CCISLSIEP AAFTVEKLVLQKYISEPVVI ELHIIITMTEVIYPVYVTLRCDSAELSGVT & \\
\hline mDGATIA & 1 & SIA $Y \mathrm{AV} \mathrm{APT}$ & \\
\hline GmDGAT1B & 17 & A ITKLVEK GEALIDTLNMDYY P & \\
\hline GmDGAT1B- & 17 & RAITKLVEKGEALIDTLNMEYPYNVTEKSIAYEIIAPT & \\
\hline VgDGATIA & 236 & R:SILNSTDKGEVEPMSSNMDYF Y DVNF|KSIVYYMVAPT & \\
\hline $\operatorname{VgDGAT} 1 \mathrm{~B}$ & 31 & TGKGEVEPMSSNMDY Y Y D NEKSIVYEMVAPT & \\
\hline AtDGAT 1 & 41 & 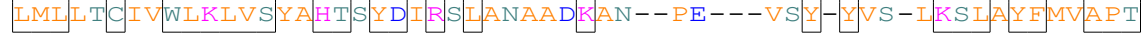 & \\
\hline $1 \mathrm{~A}$ & 71 & Se HPIKGNITYAIE & \\
\hline GmDGAT1B & 77 & TGVMGFIIEQYINPIV & \\
\hline GmDGATIB-MOD & 77 & GVMGEIIEYINPIV & \\
\hline VgDGAT1A & 296 & GEMGIIEQYINPIV| & \\
\hline $\operatorname{VgDGA}$ & 91 & FTGEMGIIEQYINPIVKNSRHPINGDFIYAIE & \\
\hline AtDGAT 1 & 294 & FTGEMGFIIEQYINPIVRNSKHPIKGDIIYAIE & 53 \\
\hline & & KTVEDYWRMWNMPV & \\
\hline GmDGAT1B & 37 & NAKTVEDYWRMWNMPV & \\
\hline GmDGAT1B-MOD & 37 & $\triangle E L I R E G D R E Y K D W W N A K T V E D Y W R M W N M P V$ & \\
\hline VgDGAT1A & 56 & AELICEGDRE EY K DWWNAQT IEE YWR TWNMPV & \\
\hline $\operatorname{VgDGAT} 1 \mathrm{~B}$ & 51 & VIKVJSVNLYVWLCMFYCFFHLWLNITAELIWWGDREYKDWWNTQTIEEYWRIWNMPV & \\
\hline AtDGAT1 & 354 & NLYVWLCMFYCFFHLWLNI AELICEGDREFYKDWWNAKSVGDYWRMWNMPV & 113 \\
\hline GmDGA & 91 & KLWAFGGIMFQVPLV & \\
\hline GmDGAT1B & & A IFHELCIAVPCHIEKTAFGGIMEQVPLV & \\
\hline GmDGAT1B- & 97 & VSA IFHELCIAVPCHMF K IWAFGGIMFQVPLV & 456 \\
\hline VgDGATIA & 16 & MSAVEHELCIAVPCHIFKFWAFIGIMQVPLV & 47 \\
\hline $\operatorname{VgDGAT} 1 \mathrm{~B}$ & 411 & YEPCRNGISKGA ILVAFEMSAVEHELCIAVPCHILKFWAFIGIMFQVPLV & 470 \\
\hline AtDGAT1 & 414 & RHIYF PCLRSKIPKTLAIIAELVSAVEHELCIAVPCRLEKIWAELGIMFQVPLV & 473 \\
\hline GmDGAT1A & 451 & PMCVLIYYHDLMNR KGKLD & \\
\hline GmDGAT1B & 457 & IGePMCVILYYHDLNR KGKLD & 4 \\
\hline GmDGAT1B-MOD & 457 & LITNYLQNKE KNSMVGNMIFW-FIESIVGePMCVTLYYHDLMNRKGKLD & \\
\hline VgDGATIA & 476 & 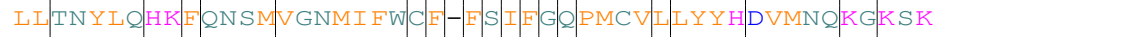 & 523 \\
\hline $\operatorname{VgDGAT} 1 \mathrm{~B}$ & 471 & LITNYLQHKE QNSMVGNMIFWCE-FSIEGQPMCVELYYHEVN-QKGKSK & 517 \\
\hline AtDGAT1 & 474 & FITNYLQEREGST-VGNMIFW-EIECIFGePMCVTLYYHDLMNRKGSMS & \\
\hline
\end{tabular}

\section{Figure S3.}


Econometric analysis of the price trends of the major plant oils vs. crude oil (petroleum) in the past

$\underline{15 \text { years }}$

Although the price trends of major plants oils and petroleum are found to move in a similar way it is not known if they move separately, or they move together. The potential price relationship among these oils was examined.

Table S2 Descriptive statistics for the major plant oils and petroleum price trends. (\$/kg) 1996 to 2012

\begin{tabular}{ccccc}
\hline Variable & Mean & Std. Dev. & Max & Min \\
\hline Palm & 0.600 & 0.265 & 1.10 & 0.24 \\
Soybean & 0.675 & 0.300 & 1.17 & 0.31 \\
Canola & 0.766 & 0.318 & 1.41 & 0.36 \\
Petroleum & 0.293 & 0.175 & 0.59 & 0.08 \\
\hline
\end{tabular}

First, the stationarity of the data set was tested (Table S2). To analyze the price/time series data the stationarity was tested, which requires that the time series values for the mean, the standard deviation, and the covariance be invariant over time ${ }^{l}$. Otherwise, the Ordinary Least Squares (OLS) regression is no longer efficient, the standard errors are understated, and the OLS estimates are biased and inconsistent ${ }^{l}$.

An Augmented Dicker Fuller (ADF) test for stationarity was conducted with the null 
hypothesis that the time series is stationary. The test is based on the t-ratio of the parameter in equation 1:

$$
\Delta \mathrm{X}_{t}=k+\emptyset t+\theta_{i} X_{t-i}+\sum_{i=1}^{n} \varphi_{i} \Delta X_{t-i}+\varepsilon_{t}
$$

Where $\mathrm{X}$ is the variable of interest, $\Delta$ is the first difference operator, $t$ captures the time trend, $\varepsilon_{t}$ is the random error term, and $\mathrm{n}$ is the maximum lag length. If we cannot reject the null hypothesis that the time series has a unit root, then we conclude the series is non-stationary.

Table S3 presents the results of the ADF test for the variables. The second column summarizes the ADF test results for levels, while the third column shows the results for the first-difference of the variables. The fourth column is the $5 \%$ critical value for determining whether to reject or fail to reject the null. All variables are non-stationary at initial levels but they become stationary after first-differencing.

Table S3 Augmented Dickey-Fuller (ADF) test results for the major plant oils and petroleum price.

\begin{tabular}{cccc}
\hline Variable & Levels & First Differences & 5\% Critical Value \\
\hline Soybean & -2.657 & $-3.898^{* *}$ & -3.600 \\
Palm & -2.343 & $-3.268^{* *}$ & -3.600 \\
Canola & -2.524 & $-4.015 * *$ & -3.600 \\
Petroleum & -3.246 & $-4.201 * *$ & -3.600 \\
\hline
\end{tabular}

Note: $* *$ represent 5\% significant level. 
Second, a cointegration test was run to check the potential causality. Based on the stationarity test, co-integration may exist since the series are integrated processes of order 1 . The Johansen cointegration test is designed to determine both the existence and the number of cointegration vectors (Table S4). The null hypothesis is that the two series are not co-integrated. We start by testing the null hypothesis of $r=0$. If it is rejected, the test for $r=1$ is performed. When a test is not rejected, the testing stops and that value of $r$ from the last test is the estimated number of cointegrating relations ${ }^{l}$.

Table S4 Johansen's test.

\begin{tabular}{cccc}
\hline Maximum Rank & Trace Statistic & $\mathbf{5 \%}$ Critical Value & Eigenvalue \\
\hline $\mathrm{r}=0$ & 119.505 & 54.64 & $\cdot$ \\
$\mathrm{r}=1$ & 51.589 & 34.55 & 0.992 \\
$\mathrm{r}=2$ & 20.077 & 18.17 & 0.895 \\
$\mathrm{r}=3 *$ & 2.209 & 3.74 & 0.721 \\
\hline
\end{tabular}

Note: * denotes the number of the rank.

A cointegration test (Table S5) yields a rank number of 3, which implies there are 3 price pairs that are cointegrated. Specifically, the soybean price and canola price, the soybean price and petroleum price, the soybean price and palm price are cointegrated, separately. Only the 
conintegration between the soybean price and petroleum price is not statistically significant. Harri,

et al. ${ }^{2}$ examined the cointegration relationship through time of some primary agricultural commodities and petroleum prices and found that soybean prices are linked to petroleum prices.

Sekhar ${ }^{3}$ also reported a relationship between the price of soybean and palm oils.

Third, the Granger causality test was applied to determine the causality among these prices based on the cointegration test. Table S5 presents the results of Granger causality test. It indicates that the soybean and canola prices influence each other. Fluctuations in the soybean price apparently caused changes in the canola price and vice versa.

Table S5 Results of Granger causality test for the major plant oil and petroleum prices.

\begin{tabular}{ccc}
\hline Null Hypothesis & Results & Significance level \\
\hline Soybean price does not Granger cause Canola price & Fail to reject & $5 \%$ \\
\hline Canola price does not Granger cause Soybean price & Fail to reject & $5 \%$ \\
\hline Soybean price does not Granger cause Palm price & Reject & $5 \%$ \\
\hline Palm price does not Granger cause Soybean price & Reject & $1 \%$ \\
\hline Petroleum price does not Granger cause Soybean price & Fail to reject & $5 \%$ \\
\hline
\end{tabular}




\section{References}

1. Enders, W., Applied econometric time series. John Wiley \& Sons: Hoboken, 2008.

2. Harri, A.; Nalley, L.; Hudson, D., The relationship between oil, exchange rates, and commodity prices. Journal of Agricultural and Applied Economics 2009, $41,501-510$.

3. Sekhar, C. S. C., Price formation in the world soybean oil market - An econometric analysis Indian Econimc Review 2008, 43, 183-204. 